\title{
Cómo surgen las preguntas
}

\author{
HOW QUESTIONS ARISE
}

\begin{abstract}
Mg. Simón Ramírez (simon@matriztica.org) Escuela Matríztica de Santiago (Santiago, Chile)
\end{abstract}
\begin{abstract}
In our living we feel and live immersed in a world of elements entities and processes that we treat as if they existed with independence from our distinguish them. From that sensoriality, as a contained belief in our nature, we lead us accepting implicit or explicitly that the act of questioning, deal with opening or discovering an independent world. That manner of thinking cannot be fundament if we in fact understand and take charge certain abstraction of our operating as human and living beings. Historically the theories around this phenomenon (cognition) is based on the belief that for one side that there isn't an objective reality but it is a mind or brain phenomena, and for the other side that exist a reality, and this we can know it, if we do thing to discover it. This article try to understand the happening of questioning, without falling in this duality, appealing to certain abstraction of our coherence as observers.
\end{abstract}

Key words: cultural biology, observer, emotions, structural determinism, cognitition

\section{Resumen}

En nuestro vivir nos sentimos y vivimos inmersos en un mundo de elementos, entes y procesos que tratamos como si existiesen con independencia de lo que hacemos al distinguirlos. Desde esta sensorialidad, como una creencia contenida en nuestra naturaleza, nos conducimos aceptando explícita o implícitamente que el acto de preguntar consiste en abrir o descubrir un mundo independiente. Ese modo de pensar no se puede fundamentar si de hecho entendemos y nos tomamos en serio ciertas constataciones de nuestro operar como seres humanos y seres vivos. Históricamente las teorías en torno a estos fenómenos (conocer) se basan en mayor o menor medida en la creencia, por una parte, que no existe una realidad sino que es una invención de la mente y/o cerebro y, por otra, que sí existe una realidad y esta es cognoscible en la medida que hacemos cosas para descubrirla. Este artículo intenta entender el suceder del preguntar sin caer en esta dualidad, apelando a ciertas abstracciones de nuestras coherencias como observadores.

Palabras clave: biología-cultural, observador, emociones, determinismo estructural, cognición

\section{Constataciones de nuestro operar como seres humanos en nuestra vida cotidiana}

Punto de partida. Sabemos que como seres humanos operamos como observadores en nuestro convivir, y que eso es posible como un ocurrir en lenguajear y conversar (Maturana y Varela 1980), por lo que el preguntar como una dinámica del operar del observador, solo es posible de entender desde ese espacio. Sabemos además que esta dinámica es abierta e infinita en nuestra convivencia, dada la naturaleza histórica recursiva del lenguaje que se sostiene a lo largo de nuestro vivir humano en las redes de conversaciones que habitamos, por lo tanto cambiando nuestro modo de convivir y cambiando los dominios cognitivos operacionalmente cerrados, ya sea imaginados, conceptuales o concretos en el 
suceder del preguntar que vivimos. Es claro además, que lo hacemos como algo habitual e inconsciente, como un aspecto más de nuestro vivir y convivir, el que sentimos como parte de una realidad independiente de nuestras distinciones, y es claro también, que lo hacemos sin ninguna reflexión sobre el sustrato operacional desde donde de hecho ocurre nuestro preguntar. Esto es, actuamos muchas veces ciegos a la dinámica o mecanismo que opera en la realización y conservación del dominio de pregunta y por lo tanto de respuesta en el cual nos encontramos, cualquiera sea este, al conservar un trasfondo de una realidad objetiva, o de un trasfondo sujeto-objeto.

En la medida que estos dominios de preguntas (y respuestas) los vivimos como surgiendo desde la nada como seres que vivimos en el lenguaje, no nos detenemos a reflexionar desde dónde aparecen o cómo ocurren, simplemente nos encontramos ya viviendo en el preguntar cuando queremos entender el preguntar o cuando nos preguntamos por el preguntar como aspectos de nuestro vivir y convivir (Maturana 2007). Así, nuestro punto de partida es la reflexión que aquí los invito: somos nosotros mismos como observadores viviendo en el lenguaje y el conversar en la simple cotidianidad de nuestros haceres, que es desde donde nos podemos preguntar por el preguntar, ya distinguiendo un mundo de objetos, procesos o fenómenos como si fuesen independientes de nuestro operar como observadores (Maturana y Varela 1984). En otras palabras, nuestro punto de partida es nuestro presente en nuestro vivir cotidiano como observadores o seres biológico-culturales (Maturana y Dávila 2008) en convivencia con otros seres biológico-culturales en la conservación del lenguajear y el conversar como nuestro modo de vivir y convivir.

No es extraño, desde esta primera constatación, el darnos cuenta que las preguntas que nos suceden tienen que ver con cómo operamos como observadores, aunque vivamos que hay un ámbito de realidad de "cosas externas" de nuestro vivir como ámbitos de explicaciones que "nos dicen algo" de un "mundo real" o de un mundo que está allí independiente de nosotros y que mediante las preguntas y respuestas logramos entender o conocer. Al vivir actualmente en una cultura patriarcal-matriarcal, las preguntas parecen estar teñidas de un carácter o de una orientación psíquica de desconfianza y competencia, haciéndolas y viviéndolas desde certidumbres y apegos sobre lo que aparece en nuestra mirada desde un mirar comparativo causal y lineal en muchos ámbitos de nuestro vivir.

Por otra parte, en la cotidianidad de nuestro presente conservamos sensorialidades y emociones de certezas, omnipotencia, control y manipulación sobre las cosas que distinguimos, incluidas las personas, y en la medida que sentimos placer al hacerlas como un modo de convivir en la realización de una cultura patriarcal-matriarcal que es nuestro modo de vivir actual jerárquico, recorremos un camino que puede destruir la propia matriz que la sostiene como la historia nos muestra, dado que se ocupan como justificaciones para nuestras acciones de discriminación y desintegración social o cultural en diferentes ámbitos de convivencia, ya sea la familia como un ámbito cultural hasta las naciones.

De este modo, tener preguntas, no es suceder trivial porque nos trasformamos alrededor de aquellas que vivimos como válidas. Todo ello revela el modo biológico-cultural o en otras palabras, las coherencias de nuestro vivir y convivir en redes de conversaciones que es donde ocurre nuestro vivir humano.

Confianza. Sabemos que los seres vivos somos entes discretos, redes de producciones moleculares que al interactuar entre ellas generan en una dinámica recursiva que constituye la misma red de producciones moleculares que las produjo. La palabra autopoiésis (Maturana y Varela 1980) evoca esta dinámica constituida como una red cerrada de producciones moleculares en las que hay moléculas que entran y se hacen parte de ella y moléculas que salen porque dejan de ser parte de ella. Sabemos que cuando 
distinguimos un ser vivo lo hacemos separándolo de un medio que lo contiene y hace posible, como su espacio de existencia o espacio relacional y que surge con él en nuestra distinción. Sabemos que un ser vivo opera como una totalidad dinámica que interactúa en su nicho en el encuentro de los elementos de este con sus bordes operacionales que son sus superficies sensoriales y que los componentes del nicho gatillan cambios estructurales en él pero no lo especifican. Por otra parte sus superficies efectoras se establecen cuando sus componentes gatillan cambios estructurales en el nicho al incidir sobre él, sin especificar lo que sucede en el nicho, o sea del mismo modo gatillando cambios estructurales en el nicho. Sabemos que el organismo y el nicho que surge con él constituyen una unidad operacional-relacional o unidad ecológica organismo-nicho, en la que existen juntos en una deriva de cambios estructurales coherentes con la conservación del vivir y convivir del organismo mientras este conserva su vivir. Además sabemos que las interacciones de un organismo en su nicho dan origen a una dinámica de gatillamientos de cambios estructurales (no semánticos), que resultan en que el organismo y su nicho cambian juntos de manera estructural y relacionalmente congruente mientras el vivir del organismo se conserva. Sabemos que como seres vivos este ámbito de encuentros estructurales es un espacio de confianza implícito en el vivir de un ser vivo donde se encuentra viviendo con los elementos del nicho que operan según su hechura en su presente, o desde un ámbito de determinismo estructural (Maturana y Varela 1980) y que su conservación regular resulta en una inercia fundamental (Maturana y Dávila 2015).

Por ejemplo, cuando usted se sentó en la silla a leer este artículo no se detuvo a ver la estructura de la silla, se sentó confiando en su hechura, a menos que la silla se haya roto últimamente y sienta que no está del todo regular en su estructura como lo solía ser (en ese caso confía en la nueva estructura de la silla, la silla que se rompió). Por otra parte, si usted, lectora o lector, siente que este artículo no tiene final o no va hacia ninguna parte probablemente no lo seguirá leyendo, más aún, si usted siente-piensa desde un espacio de su vivir como todo lo que le pasa en su presente, que este es un artículo de filosofía y no uno de biología y usted busca uno de biología, usted se sentirá invitado a abandonar su lectura desde un espacio sensorial-operacional-relacional que usted vive como válido. Lo mismo sucede con una mariposa que si llega a sentir peligro (mal-estar) cambiará su camino (vivir) en donde se encuentre con uno desde alguna dimensión sensorial de su vivir que viva desde ella, como no miedo, amenaza o confianza en la conservación de su vivir.

En otras palabras, los seres vivos vivimos como válido todo lo que vivimos en el momento de vivirlo, y lo que hacemos momento a momento, es comparar consciente e inconscientemente experiencias en un espacio de determinismo estructural. De este modo, si vivimos como válida una experiencia en referencia a otra de la cual no dudamos como válida, la llamamos percepción. A su vez, si vivimos como válida una experiencia en referencia a otra de la cual dudamos y la vivimos como inválida cuando comparamos una experiencia con la otra de la cual no dudamos de su validez, la llamamos ilusión o error (Maturana 2005). En estricto rigor estamos siempre operando con las coherencias de nuestra experiencia en algún dominio de determinismo estructural, que es un ámbito operacional relacional y sensorial, moviéndonos o deslizándonos (Maturana y Mpodozis 2000) en un presente en continuo cambio con las coherencias de nuestra experiencia abierto a un cambio en nuestra experiencia transformando, de ese modo, aquello que podemos vivir como válido.

Podemos apreciar que nos movemos en completa confianza operacional consciente o inconsciente según lo que vivimos como válido, de acuerdo a nuestra dinámica sensorial momento a momento en el dominio de coordinaciones de coordinaciones de haceres, sentires y emociones que el lenguaje es, como una dinámica relacional (Maturana 1988). Lo anterior ocurre en un presente en continuo cambio de carácter histórico recursivo en el sentido de que el vivir y convivir se transforma momento a momento de acuerdo 
al momento previo. Fluir que, en el espacio humano de coordinaciones de coordinaciones de haceres, sentires y emociones, es la fuente de los mundos que vivimos como distinciones de las cuales podemos hablar, ver, tocar, oír, como observadores en el lenguaje.

Sin duda esta confianza implícita en la mayor parte de nuestro vivir es inconsciente, no se requiere que se "piense" ni que se expliquen las coherencias con el medio y cuando se interrumpe un dominio de determinismo estructural, buscamos encontrar otro.

Historia. Sabemos y entendemos que el organismo y su nicho constituyen una unidad, sabemos que el organismo y el nicho cambian juntos congruentemente en un fluir de interacciones estructurales, y que este flujo de interacciones constituyen un proceso de co-transformaciones o acoplamiento estructural (Maturana 1988). Sabemos que el curso que sigue ese proceso de acoplamiento es guiado por la sensorialidad del ser vivo, evocadas como preferencias y deseos en el espacio conductual-relacional. Por ejemplo si nuestro deseo es ser estudiantes, todo se transformará en torno a la conservación de ese deseo, hasta que ese deseo se interrumpa y dejemos de conservar la dinámica sistémica relacional que surge en nuestro vivir y convivir.

Es así como en cualquier circunstancia de nuestro vivir y convivir estamos en continua trasformación en torno al espacio sensorial y relacional según lo que conservamos. Y lo que distinguimos como un resultado de ese vivir y convivir, lo es siempre de acuerdo a la historia de transformaciones en torno al cual se conservó o resultó conservado nuestro vivir y convivir como parte de una deriva (Maturana y Mpodozis 2000) y que es un espacio disjunto del proceso que le da origen.

Es cierto también que en muchas ocasiones adscribimos relaciones causales locales al momento de entender la ocurrencia o coherencia de cierta circunstancia o fenómeno, parándonos desde un lugar explicativo que vive como válido consciente e inconscientemente el que se pueda especificar conductas desde lo que llamamos traspaso de información, dejando en ese instante de ver la riqueza sistémica histórica de transformación congruente de cualquier sistema en un ámbito también sistémico, en donde existe siempre una co-deriva de transformación de muchas más dimensiones de las que podemos dar cuenta. En otras palabras, en un ámbito humano nos vemos desde una co-deriva histórica como un caminar en donde el otro(s), la otra(s) o lo otro(s) se vuelven parte recurrente y recursiva del medio y del nicho de uno, y por ello somos como somos en el presente de acuerdo a una historia de trasformación atemporal en una co-deriva social-cultural de nuestra unidad ecológica organismo-nicho.

Es la conservación en un presente de deseos o preferencias lo que permite o abre el espacio a conductas posibles en donde puede cambiar toda nuestra estructura, conservándola así mismo como arquitecturas dinámicas sistémicas recursivas internas desde la conservación básica de la realización de nuestro vivir o autopoiésis en coherencias con el espacio que las hace posible. Es por esto que nuestro cambio corporalsensorial, en tanto ser humano, siempre es coherente con nuestro modo de vivir cultural en redes de conversaciones. Así podemos ver que el espacio humano en el lenguajear se sostiene en redes de conversaciones, en que nuestro cambio estructural ontogénico cambia en torno al fluir histórico de las redes de conversaciones que conservamos en nuestro vivir y convivir.

Dominios cognitivos. Sabemos que como observadores decimos que una persona sabe o conoce y estamos diciendo que ella o él se conduce de una manera que consideramos adecuada porque satisface algún criterio de validez que ponemos en nuestro observar-escuchar al aceptarla (Maturana 1988). Por lo mismo, cuando decimos que no sabe (sabemos), decimos que en ese instante pensamos o vivimos como 
valido que él o ella no se conduce de manera adecuada según lo que consideramos es una manera adecuada de hacerlo. Así al hablar de lo que sabemos hablamos de diferentes clases de configuraciones de sentires $y$ haceres que aceptamos como fundamentos operacionales-relacionales de manera consciente e inconsciente la mayor parte del tiempo y en ese espacio también aparecen errores, incertidumbres y dudas. ¿Cómo es que ocurre esto? Una aseveración que involucra una distinción de saber o conocer en cualquier contexto se basa solamente en las coherencias operacionales que el observador vive como válidas y no de una realidad independiente de él o ella. En este sentido el conocer hace referencia a la conducta aceptada como efectiva en un dominio particular que el mismo observador especifica desde alguna distinción como válida o legítima. Un dominio de conocimiento o cognitivo es un dominio de haceres, distinciones o acciones que un observador (un conjunto de observadores) acepta como legítimo en un dominio particular, y se configuran como dominios particulares de coherencias sensoriales-operacionales-relacionales en el vivir y convivir de quien las realiza y vive. Si lo miramos de otra manera, podemos decir que conocer es configurar desde una relación (encuentro) con el medio desde el vivir del ser vivo un dominio de acciones (conducta) cerrado de coherencias con la experiencia, así el cocinar, el jugar, el ser ciudadano, el ser profesor, el ser esposo, etc., son diferentes dominios cognitivos que se entrelazan o intersectan en nuestra corporalidad.

Nos podemos dar cuenta también que un dominio cognitivo también es un dominio racional en el lenguaje (operación de distinción) en la medida que es un dominio de abstracciones en un ámbito de distinciones de las coherencias sensoriales-operacionales-relacionales (Maturana y Dávila 2015) en el vivir y convivir especificado desde los gustos y preferencias (sentires íntimos) que da forma a los criterios de validación que un observador usa para aceptar acciones como acciones efectivas en el espacio del explicar. Por ejemplo la explicación sobre evolución puede tener distintos dominios racionales (explicaciones) como observadores que conserven criterios de validación desde sus gustos y preferencias. En este sentido todos los dominios cognitivos racionales-explicativos tienen igual legitimidad en su constitución, pero no son igualmente deseables en la convivencia. Hay tantos dominios cognitivos como diferentes criterios de validación o aceptación que un observador usa para aceptarlos como adecuados en un cierto ámbito.

El vivir en el lenguaje (lenguajear) conservado en el conversar como el modo de vivir humano en coordinaciones de coordinaciones consensuales de haceres, sentires y emociones, es una co-deriva estructural, en el sentido que es un proceso que no ocurre en el vacío, ocurre en la interacción recursiva de al menos dos seres humanos. El lenguaje como generador (no constructor) de realidades en la convivencia se constituye desde la concretitud de los haceres en coordinaciones de haceres, por lo tanto lo que distinguimos y de lo cual podemos hablar o no, surge en una matriz biológica (autopoiésis, corporalidad sentires) -cultural (relaciones en redes de conversaciones) y se conserva en un presente en una matriz de la misma naturaleza, en la generación y conservación de dominios cognitivos desde las redes de conversaciones que las definen.

En lo humano el vivir en el lenguaje (lenguajear) y el conversar es la fuente de los espacios cognitivos explicativos racionales, que involucra los criterios de validación (Maturana 1988) que ponemos desde nuestros sentires y emociones en nuestro vivir de manera inconsciente la mayor parte del tiempo, y que pasan a ser referentes operacionales en la convivencia que modulan nuestros haceres posteriores que aparecen en nuestro vivir. Las explicaciones son reformulaciones de la experiencia con elementos de la experiencia (Maturana 1988), que se constituyen como procesos o mecanismos concretos u abstractos tales que si los dejamos operar darían como resultado lo que quisiéramos responder. En este sentido el que validemos una explicación como explicación legitima en nuestro vivir, tiene que ver con los criterios 
de validación que ponemos desde nuestros sentires y emociones y que pasa por lo que se desea conservar desde un gusto o preferencia no siempre consciente.

Bajo lo anterior, los distintos espacios cognitivos surgen en una comunidad de personas que aceptan criterios de validación que constituyen acciones o conductas (explicaciones y haceres) adecuadas dentro de su comunidad desde la conservación de gustos, preferencias, sensorialidades comunes, etc. Nosotros seres humanos podemos vivir muchos distintos dominios cognitivos racionales como dominios de coherencias con la experiencia, como redes de conversaciones y nos movemos cambiando uno de otro desde nuestras emociones y sentires. El que vivamos dominios cognitivos como dimensiones conductuales y racionales de nuestro vivir y convivir es el resultado de la congruencia sensorial-operacional-relacional organismo-nicho en donde el medio (nicho) y organismo cambian juntos guiado desde la propia sensorialidad (sentires) del organismo, en este sentido nunca estamos fuera de lugar o no congruentes (incongruencia) con nuestro medio-nicho.

Sistema nervioso. Imaginemos que vamos en la calle e intentamos buscar nuestro auto que lo hemos estacionado. Imaginemos que justo vemos otro auto igual al de nosotros desde una perspectiva, ¿cómo sabe uno qué auto es el suyo? Lo que una persona hace es ver algún detalle que los diferencie. Es así que la persona que lo busca encuentra justo una marca cerca de la puerta del piloto que el otro no lo tiene y lo identifica como el suyo, ¿qué pasa? El medio no le dice ni le especifica nada a la persona porque los seres vivos somos sistemas determinados en nuestra estructura, de hecho sabemos que el sistema nervioso no opera con elementos del medio, sino que opera cerraderamente como cambios de relaciones de actividad entre los componentes que lo componen. Es el organismo como un todo el que se encuentra con un medio que lo hace posible pero que no especifica su operar, en un encuentro que es de naturaleza estructural no semántico. $Y$ un observador ve como conducta a ese encuentro como acciones sobre un entorno o medio pero que en el operar del sistema nervioso componente del organismo corresponde a un fluir en correlaciones senso-efectoras coherentes con la historia del ser vivo (ontogenia), por lo que el sistema nervioso no opera con símbolos o representaciones, sino que estos surgen como distinciones (objetos) en el lenguaje. Lo que el sistema nervioso hace es operar distinguiendo configuraciones (operaciones) relacionales que se establecen inconscientemente desde el vivir biológico-cultural en el caso humano (espacios cognitivos) que en el lenguajear se viven como mundos de objetos, pero que son configuraciones senso-efectoras en el vivir del organismo y cambios de configuraciones de relaciones de actividad en el sistema nervioso. Así inconscientemente a través de una historia de transformación en redes de conversaciones como espacios cognitivos (haceres), la persona distingue diferentes configuraciones como auto, color, marca de automóvil, ruedas, vidrio polarizado, calle, etc., que en el sistema nervioso como componente del organismo pasan a ser cambios de relaciones de actividad en un solo dominio, que es coherente de nuevo con la historia de ese observador que busca el auto, de modo que todas estas configuraciones se intersectan en la corporalidad de la persona de manera inconsciente hasta que una marca cerca de la puerta del piloto le gatilla la diferencia en ese presente sensorial, pues todas las demás se viven como "iguales". Este fenómeno ocurre inconscientemente y nos trasformamos en la convivencia generando, conservando y transformando configuraciones relacionales en la realización de nuestro vivir y convivir. Es a esto a lo que llamamos aprendizaje y no a un modo de captar algo del medio.

Pensemos ahora en el ejemplo de una llave y cerradura, la puerta resulta abierta dado que en la relación la llave gatilla un cambio de configuración en la cerradura, si la cerradura cambia, la llave ya no abre la puerta (conducta), por lo tanto la llave (medio) solo gatilla algo en la cerradura (ser vivo). Lo mismo pasa con nosotros, pero el inmenso número de cerraduras que ocurre en nuestro sistema nervioso dependen 
del presente de trasformaciones históricas de nuestro vivir desde nuestro naturaleza filogénica y ontogénica, que cambian, se trasforman y se conservan dinámicamente en un presente en continuo cambio.

\section{Respuesta}

¿Qué hacemos y qué ocurre cuando comenzamos a vivir inmersos en dominios de pregunta-respuesta como lo hacemos ahora? No tener una respuesta (explicación) es un modo de tener una respuesta del mismo modo que cuando decimos no sé, porque es una manera de convivir (conducirse) cuando se acepta una pregunta como válida en algún momento y circunstancia de nuestro vivir; y es un modo de conducirse o deslizarse en las coherencias sensoriales-relacionales-operacionales en la confianza en el dominio particular en el cual queremos conservar (conscientemente o no) algún aspecto de nuestra sensorialidad. Somos el presente siempre de una historia biológica-cultural y de una epistemológica-cognitiva en la medida que lo único que hemos conservado es nuestro vivir y los espacios relacionales (adaptación) bajo distintas identidades o dominios cognitivos que esta pueda adoptar en nuestra unidad organismo-nicho.

Reitero, la conservación depende del espacio de preferencias del ser humano, desde un ámbito sensorial siempre en continuo cambio. Los espacios que surgen desde nuestras preferencias, gustos o deseos, duran lo que duran nuestras ganas de estar ahí, y se terminan cuando perdemos las ganas, la preferencia o el gusto (la sensorialidad) que resulte en estar ahí. La mayoría del tiempo no somos consciente de este suceder biológico, lo mismo con las respuestas, las cuales aceptamos desde un criterio de validación aprendido (modo de relacionare interpersonalmente) desde un espacio de trasformación histórico en que las aceptamos porque nos gustan desde algún espacio sensorial de confianza operacional.

Para el operar de un observador consciente de que el mundo que vive surge con su vivir y convivir, lo que explicamos no es lo que usualmente llamamos fenómeno o proceso como algo independiente de nuestro operar o de nuestro vivir. Lo que explicamos es algo que nos pasa en nuestro distinguir o en el acto de distinguir lo que nos sucede en el lenguaje. Por lo tanto, lo que hacemos es explicar nuestra experiencia con las coherencias de nuestra experiencia. Si no tenemos consciencia de que estamos distinguiendo algo desde que nos pasa ni siquiera hablamos de ello, y no participa del mundo como redes de conversaciones. Las bacterias no participaban en el pasado como explicaciones de lo que llamábamos enfermedades o infecciones, ahora (últimos 200 años) con la expansión de nuestro dominio cognitivo en la expansión de la tecnología (microscopio) las bacterias como operación de distinción (dominios cognitivos racionales) surgieron como una explicación (respuesta) válida por la pregunta de cómo surgen las enfermedades, esto es, surgió un nuevo dominio de explicaciones o cognitivo (haceres).

La descripción de la experiencia no reemplaza a la experiencia. Nunca lo hace. El que diga que esté asustado no hace que esté asustado. La descripción es secundaria y ocurre como tal en el dominio de la reflexión en el lenguaje. Pero los seres humanos vivimos en redes de conversaciones en distintos espacios de explicación que vivimos como diferentes dominios cognitivos y a su vez como distintas coherencias con la experiencia que las definen. Más aun, de acuerdo a lo mencionado, un dominio cognitivo desde nuestra corporalidad es un fluir de correlaciones senso-efectoras en la continua realización de la autopoiésis molecular en un espacio relacional que surge de esa matriz de correlaciones senso-efectoras.

De este modo, las preguntas aparecen desde un dominio sensorial como un reordenamiento de nuestra sensorialidad dentro de nuestra unidad multidimensional organismo-nicho. Este reordenamiento resulta en correlacionar coherentemente nuestros dos espacios de existencia: el de nuestra fisiología-sensorial 
con nuestro espacio relacional o conductual. Esta correlación operacional, sensorial y relacional ocurre como parte de la conservación de nuestra unidad organismo-nicho, por lo tanto es un reordenamiento de nuestra sensorialidad en la conservación congruente en nuestra unidad organismo-nicho.

La sensorialidad ocurre en un presente de nuestro vivir y convivir histórico en redes de conversaciones, que van conservándose o no pero que van dando forma y estructura a nuestra sensorialidad presente. En estas redes de conversaciones aprendemos los múltiples espacios cognitivos que se viven en nuestra corporalidad como configuraciones operacionales-relacionales, y las configuraciones que aprendemos son muchas más de las que damos o podemos dar cuenta, así incontables veces nos sorprendemos por cierto resultado de nuestro actuar o el de los demás, como si viniesen de la nada o si en ese minuto pasó algo inesperado, quedando oculta la dinámica de trasformación congruente histórica del sistema nervioso, la corporalidad, el organismo y el medio.

Por otra parte la consciencia del preguntar-responder solo es posible en el lenguaje como un modo de convivencia que trae a la mano entidades como operaciones de distinción y relaciones entre ellos en el que surge un mundo de "cosas" como un modo de convivencia o dominios cognitivos abstractos racionales entre seres humanos y en el que hace sentido hablar de ellos de acuerdo a una historia de co-deriva de deseos comunes.

El resto de los animales se mueven desde su sensorialidad en la realización de un nicho y una unidad ecología organismo-nicho, coherente con la historia de deriva ontogénica y filogénica. Ellos no reflexionan sobre cómo se comportan pues no viven en el lenguaje en donde el yo surge en el lenguaje (Maturana 2005). Por ejemplo en el nadar o volar, ellos no nadan o vuelan, estas son nociones que proponemos para explicar el cómo se mueven (cambio en su posición relativa en referencia a otra en cierto contexto). En los diferentes medios que surgen en nuestras operaciones de distinción con propiedades y características estructurales y relacionales, el nadar y volar existen para nosotros seres humanos que viven en la generación de mundos en el lenguaje. Si los animales pierden la confianza, como vimos, en las coherencias operacionales desde un dominio sensorial o de sentires íntimos en los espacios cognitivos que viven, pérdida de confianza que un observador ve como cambio en el modo que se realiza la unidad organismonicho, buscan recuperar, crear o conservar desde el reordenamiento de su sensorialidad otro(s) dominio(s) cognitivo(s) en un espacio de determinismo estructural. Un observador puede ver esto como duda, inconformidad, desconfianza o incongruencia en su modo de conducta, pues la regularidad en algún dominio de determinismo estructural de origen histórico se interrumpe. Cuando esta regularidad se pierde, se reordena la sensorialidad en su unidad ecológica organismo-nicho, o sea, se transforma la manera en que se realiza la unidad ecológica organismo-nicho, y abre espacio para la reformulación de su experiencia con elementos de su propia experiencia (explicación, respuesta o acción). Como los dominios cognitivos no tienen que ver con los elementos sino con la operatividad o mecánica, un espacio cognitivo surge en la unidad ecológica organismo-nicho que resulta en una nueva configuración regular desde el reordenamiento de nuestra sensorialidad, que a su vez aparece desde la captación de una configuración relacional en el espacio conductual en nuestra unidad ecológica organismo-nicho. Siguiendo el ejemplo del auto, la pregunta surge al reordenar la sensorialidad en la unidad organismo-nicho (personaautomóviles) desde la desconfianza (se busca la consistencia del determinismo estructural) de cuál es su automóvil en la regularidad en donde se conserva la posesión de su automóvil. Así una dimensión de las muchas que vivimos en el presente dejó de ser regular desde nuestra configuración sensorial, y se busca, de manera consciente o no un nuevo ámbito de confianza sensorial-cognitivo en el determinismo estructural, y se busca reformulando nuestra experiencia. 
Así la persona capta una configuración novedosa, (busca) la pequeña marca, pudiendo ser otra, por ejemplo la posición del auto en referencia a la esquina de la calle, de modo que al confiar en esa configuración (experiencia) se establece o recupera en este caso esa incongruencia (en ese dominio) perdida como un ámbito de determinismo estructural.

En otras palabras, dado que nos movemos desde nuestra sensorialidad (sentires íntimos) generando espacios relacionales o espacios cognitivos concretos o abstractos (racionales), en la conservación de nuestra unidad ecológica organismo-nicho, surgen lo que un observador llamaría vacíos en su vivirescuchar-ver, o en su multisensorialidad, en fin, en un sentir en que se siente él o ella operando en un domino de no confianza ante una encrucijada o pérdida de regularidad sensorial en un espacio recursivo (la búsqueda del auto solo es posible sobre una operación anterior que es el estacionar el auto y así hacia atrás) sobre lo que él o ella contempla como un medio independiente de él o ella, de manera que surge una incongruencia en su vivir relacional-operacional desde esa configuración sensorial en que no resulta un dominio cognitivo conceptual u operacional que dé cuenta de su continuar vivir en confianza que él o ella trae a la mano en un dominio cognitivo conceptual.

Surge la posibilidad de realizar una pregunta en fluir de nuestro vivir y convivir, desde el reordenamiento de nuestra sensorialidad en alguna dimensión sensorial íntima de desconfianza e incongruencia en el determinismo estructural, en nuestra unidad organismo-nicho desde nuestro vivir biológico-cultural con nuestros criterios de validación (experiencia) incalculables, en el espacio ya sea consciente o autoconsciente en el lenguaje y el conversar en el deseo de recuperar conservar (confirmar) o crear un dominio de coherencias sensoriales-operacionales-relacionales perdidas o en la creación de un nuevo dominio cognitivo humano coherente con nuestra historia en la conservación de nuestra unidad organismo-nicho que ocurre en redes de conversaciones en donde somos el presente de esa historia. Por ejemplo si un niño comienza a vivir en el lenguaje en la infancia se encuentra viviendo que él o ella es el centro de las reflexiones o distinciones que hace, aparece el yo (Maturana y Gerda Verden-Zöller 2008), una nueva dimensión recursiva en el lenguaje, en la unidad organismo-nicho de su vivir, y puede aparecer la pregunta por como nacía, quizás la madre le comenta que nació vía la cigüeña, al crecer y cambiando el dominio de experiencias en las redes de conversaciones que habita, la pregunta por cómo nació cambia o se conserva, es posible ahora la pregunta ¿de dónde viene la cigüeña?, pues las coherencias experienciales de la unidad organismo-nicho del niño cambia, y así recursivamente.

El que aparezcan preguntas desde un espacio sensorial y cultural no es un tema trivial porque la clase de preguntas que aparezcan como un reordenar nuestra sensorialidad definen el espacio de la respuesta o camino posible. Si nos preguntamos por un ¿cómo? queremos un mecanismo como respuesta. Si nos preguntamos por un ¿por qué? queremos o escondemos un principio como respuesta. Las distintas palabras que tenemos para evocar una pregunta son distintas maneras de evocar nuestro fluir epistemológico que surge de las redes de conversaciones que hemos aprendido y que conservamos en nuestra convivencia cultural.

Nuestros mundos culturales no florecen de cualquier manera, no surgen desde la casualidad, azar y caos. Aunque nos pueden parecer sorprendentes, exóticos, raros o incomprensibles, son siempre expansiones de nuestras coherencias como observadores, es así que la naturaleza de la transformación resulta tan importante pues si cambian nuestras coherencias, surgen nuevos ámbitos de coherencias como expansiones de las anteriores, y un nuevo espacio de preguntas puede aparecer. En nuestro espacio humano cada recursión en nuestro conversar abre la posibilidad de un dominio nuevo de distinciones y de 
coordinaciones de haceres sentires y emociones que constituye la posibilidad de un vivir y convivir en un preguntar completamente nuevo.

En otras palabras, todo cambia en torno a las redes de conversaciones que conservamos, los criterios de validación también se transforman de manera inconsciente, por lo tanto lo que vivimos como válido en una circunstancia la podemos vivir como no válida en otra y esto está pasando siempre, incluso si no reflexionamos sobre lo que decimos y hacemos. Por ejemplo si pensamos (vivimos como válido) que este artículo no dice nada o es basura generamos un mundo de acuerdo a eso, de modo que viviremos el basurear este artículo como un aspecto válido de nuestro vivir y convivir y en donde ciertas cosas pasaran y otras no, o algunas tendrán presencia al leer otros libros en relación a esto, o no serán parte del mundo que habitamos.

\section{Reflexión Final}

Interesantemente las preguntas y respuestas no son, ninguna de ellas, una condición de existencia para la conservación de nuestro vivir como unidades autopoieticas, pero sí lo son para nuestro vivir humano en el espacio relacional en donde toman lugar como un modo de operar en redes de conversaciones que cambian y conservan fluidamente el mundo en que vivimos o sea para nuestro distintos habitares en los mundos relacionales que aparecen con nosotros, mas no para el resto de las dimensiones del vivir que pertenecen al ámbito de la modulación conductual que tienen presencia en el dominio relacional y que a su vez puede resultar en un cambio del dominio fisiológico-sensorial significativo o no, pues el lenguaje tiene la concretitud de los haceres. Ello, aunque pueda cambiar la forma en la que nos encontramos a través de las preguntas y respuestas con nosotros y/o con los demás generando mundos en la convivencia desde nuestra corporalidad como el ámbito en que se entrecruza todo.

Uno no responde para encontrar una verdad o una realidad o para revelarla o traerla a la luz desde un trasfondo oculto o de difícil acceso. Lo que hace es revelar o traer a la mano desde nuestra matriz biológica-cultural una dimensión de nuestro sentir-emocionear-pensar-actuar, coherente con cómo hemos vivido nuestro vivir. Por esto es tan fundamental la educación o cómo nos trasformamos en especial los niños y niñas, pues ellos no pueden reflexionar como lo podemos hacer los adultos, y por esto es tan fundamental para nuestra convivencia que seamos conscientes y responsables de qué vivimos como válido y en qué espacio sensorial-emocional está ocurriendo, de eso depende que ellos puedan hacerlo en algún momento. ¿Conservamos nuestras certezas, arrogancias, solidaridades, miedos, angustias, etc.?

Las preguntas-respuestas no tienen que ver con lo distinguido tiene que ver con nuestro hacer, con cómo vivimos la generación de los mundos como espacios conductuales de encuentro con un medio, en la realización de un nicho biológico-cultural que es el modo de conservar un vivir cultural desde la confianza que implica nuestras coherencias operacionales experienciales en donde de hecho está nuestro bien-estar, nuestros paraísos, nuestro mal-estar y nuestros infiernos.

Por último, es claro desde lo que hemos visto, que ninguna pregunta como experiencia es trivial, pues surge como un modo de reorientar nuestra sensorialidad y por lo tanto de reorientar lo que ocurre en nuestro espacio relacional, esto es, como un modo de recuperar desde ese reordenamiento las coherencias sensoriales-operacionales-relacionales de nuestro vivir y convivir en algún ámbito de nuestra existencia, con mayor o menor presencia de acuerdo a cómo lo vivamos y qué queramos en algún ámbito. Por lo tanto desde donde nos ocurran las preguntas será el espacio posible de las respuestas que nos encontremos y aceptemos como modos de reformular nuestra experiencia con elementos de nuestra 
propia experiencia. Así como explicamos las coherencias sensoriales-operacionales-relacionales de nuestro vivir-convivir con las coherencias sensoriales-operacionales-relacionales de nuestro vivir-convivir, nos encontramos con que muchas veces creemos que explicamos las coherencias de nuestro vivir con las coherencias sensoriales, operacionales y relacionales de un dominio trascendente, lo que no ocurre bajo ningún modo.

La dinámica por lo tanto de nuestro vivir y convivir biológico-cultural es el fundamento generador de nuestro vivir y convivir diario, y nuestra gran dificultad para entender el mundo que habitamos desde su amplia diversidad como seres sociales. Es la conservación y realización de nuestra cultura patriarcalmatriarcal que actualmente vivimos, cultura centrada en la apropiación fundamentalmente (de todo lo imaginable), en el control, en el apoderamiento, en la inseguridad, competencia, victimización, el miedo, falta de interés genuina por los otros y en la adicción a ser servidos, etc., todos espacios emocionales que generan una convivencia centrada en preguntas y respuestas reduccionistas y lineales no sistémicas, ciegas a un mirar que interconecta elementos y procesos (o sistémico), y esto no es trivial pues guiará los espacios de nuestra convivencia humana. ¿Qué espacio emocional abre un preguntar y mirar sistémico?

Nota. El autor fue Coordinador del Magíster en Biología Cultural de la Universidad Mayor (Chile) entre 2012 y 2015.

\section{Bibliografía}

Maturana, H. 1988. Ontología del conversar. Revista de Terapia Psicológica 7(10): 15-23.

Maturana, H. 2005. The origin and conservation of self-consciousness: reflections on four questions by Heinz von Foerster. Kybernetes 34(1-2): 54-88. doi: 10.1108/03684920510575744

Maturana, H. 2007. Systemic versus genetic determination. Constructivist Foundations 3(1): 21-26. www.univie.ac.at/constructivism/journal/3/1/021.maturana

Maturana, H. y Varela, F. 1984. El árbol del conocimiento: las bases biológicas del conocer humano. Santiago: Editorial Universitaria.

Maturana, H. y Varela, F. 1980. Autopoiesis and cognition: the realization of the living. Boston: D. Riedel Publishing Co.

Maturana, H. y Mpodozis, J. 2000. The origin of species by means of natural drift. Revista Chilena de Historia Natural 73(2): 261-310. doi: 10.4067/S0716-078X2000000200005

Maturana, H. y Dávila, X. 2008. Habitar humano: en seis ensayos de biología-cultural. Santiago: JC Sáez ediciones.

Maturana, H. y Verden-Zoller, G. 2008. Origins of humanness in the biology of love. Exeter: Imprint Academic.

Maturana, H. y Dávila, X. 2015. El árbol del vivir. Santiago: MVP editores.

Recibido el 4 May 2015

Aceptado el 6 Jun 2015 